\title{
Optimization of a Production Process from Demand and Number of Defective Units through Diffused Logic and Genetic Algorithms
}

\author{
Christian A. Mejía Ramírez, Martin Montes Rivera \\ Universidad Politécnica de Aguascalientes, Aguascalientes, \\ Mexico \\ mc170003@alumnos.upa.edu.mx, martin.montes@upa.edu.mx
}

\begin{abstract}
Production planning and scheduling are important activities in enterprises since they allow maximizing productivity by efficiently managing financial, material and human resources for achieving production objectives, avoiding excessive or insufficient stock material. Production time and faulty pieces are common analyzed variables with different mathematical techniques applied to improve production processes, nevertheless, these methods can obtain solutions with variable quality depending on the given input data. An alternative for classical methodologies that does not require complex mathematical models or high precision data in an environment of imprecision and uncertainty is fuzzy logic, but even with this technique, tuning inference systems demand long time for achieving good results in a production process, an alternative is to hybridize this algorithm together with optimizing algorithms for adjusting the required parameters. Genetic Algorithms (GA) are metaheuristic optimizing systems for exploring possible solutions to well-structured problems, which have shown good results in the parametrizing of complex problems, using natural selection principles. In this paper is proposed a fuzzy inference system that allows planning and scheduling production using a Mamdani inference system tuned with a GA.
\end{abstract}

Keywords: planning of production, fuzzy, genetic algorithms, optimization.

\section{Introduction}

\subsection{Description of Problem}

Nowadays, the planning of production is one of the major problems faced by the managers and administrators of this process; among the variables that can be found in the problem are those corresponding to production levels, the supply chain, demand, 
and capacity in stock, defective units, among others. The main objective of the production is the reduction of the total costs; however, in many situations there may be others, such as minimizing defective units in the process; or, to maximize production levels.

For example, one of the main objectives regarding the problem of the supply chain is the minimization of the costs of controlling the flow of materials among suppliers, industries, warehouses and users. With regard to the economic quantity of order in a company, it is primarily sought to minimize the inventory costs from the demand that is had, usually in regular periods of time. Another important problem is the aggregate planning, which consists in the determination of the quantity of production and its development of time to certain term.

In production planning there are several factors that are not foreseen when they are carried out, among which are the defective units that can be had, this problem can delay the delivery of products to meet the demand; or, not anticipate in time to fulfill the demand; that is why, by programming a diffuse inference machine, the best decision to be made is sought with respect to the level of production that one wants to reach, when there is a certain demand and a quantity of defective units.

\subsection{Related Works}

From its very beginnings fuzzy logic have been implemented for the solution of several industrial problems, where in diverse situations there are scenarios of imprecision and uncertainty; however, to such problems a decision must be made in the most appropriate way possible. In the article by Kaharam, Gülbay and Kahraman [11] several problems that have arisen in the industry are mentioned, among them the planning of the production; However, no mention is made of the defective units that may be reached in the production process, this is a variable that cannot be overlooked in planning, since this can generate delays with compliance with the satisfaction of the demand.

In [12], a diffuse model is applied to the planning and programming of steel production; This model had great benefits in terms of reduction of stock and storage costs, increases safety when making decisions and improves quality; however, the time factor to be used in the process must be taken into account, since in many situations it does not depend directly on the manager; the malfunction of a plant or machinery that causes defective units in most cases, is a mechanical problem. In the present article this variable is not omitted, in fact this can be implicit in the demanding and defective units, since from these variables we can determine the level of production (greater or less than stable), that is required, and with it, to know the operation time of the plant.

On the other hand, the investments that handled the planning of the production are important since it is intended that the costs be the minimal possible, in the article by Kahraman and Tolga [13], diffuse logic techniques are proposed to choose the most appropriate way to carry out investments in planning processes; however, not always the failures in the process are directly attributed to the manager of the planning, in different situations it depends on the capacity and effectiveness of the production plant. In this article, costs are not directly minimized, but if a strategy for cost minimization 
is given in the action of production based on the demand and the defective units, then costs would be minimized.

Another contribution in this research is the genetic algorithms application, since they were used in the tuning of membership functions. These can carry out a better proposal of the belonging functions. In the article Gladkov, Gladkova and Gromov [14] it is proposed a solution to the planning of production processes by means of fuzzy logic and genetic algorithms, very similar to what is proposed here, but Gladkov article does not define variables such as defective units and the demand for make the decisions.

\subsection{Theoretical Framework}

The optimization applications are countless. Each process is feasible and optimized. There is no company that does not involve optimization problems within its activities. Many of the industrial processes can be formulated as optimization processes, such operations are presented in minimizing the time invested for the accomplishment of a task, the cost of elaboration of a product and the risk in an investment, or they can also be presented in the maximization of profits, product quality and efficiency of a device, among others.

The great part of the problems of optimization with practical implications in the industrial engineering are very complex and difficult to solve, among them we can mention the maximization of the production in a production line in such a way that the demand is satisfied without defective units. Such problems cannot be solved in an exact manner using classical optimization methods; however, under these circumstances the fuzzy logic methods and genetic algorithms have been consolidated as an alternative solution, because they do not require high precision information, only very general information given by an expert.

Production planning policies must balance production costs and losses for defective units, with the costs of not being able to meet an order on time. Below there is a model of production planning based on the demand that is held in a certain period and the defective units that can be present.

A diffuse set $\mathcal{A}(1.1)$, is defined as an assembly that can contain elements in a partial way; that is, the property that an element belongs to the set can be true with a certain degree of truth, said degree of truth is given by a function $\mu_{\mathcal{A}}: U \rightarrow[0,1]$, where $U$ is a universe and $[0,1]$ is the closed interval with ends 0 and 1 .

This definition associates each element $x \in A \subseteq U$ with a real number $\mu_{\mathcal{A}}(x)$ in ranges $[0,1]$, which is assigned to $\mathrm{x}$. Then the diffuse set $\mathrm{A}$ can be written as in (1):

$$
\mathcal{A}=\left\{\left(x, \mu_{\mathcal{A}}(x)\right): x \in A, \mu_{\mathcal{A}}(x) \in[0,1]\right\} .
$$

Let $\mathrm{A}$ and $\mathrm{B}$ two diffuse sets with $\mu_{\mathcal{A}}$ and $\mu_{\mathcal{B}}$ membership functions, respectively: The intersection operation (2) of $\mathcal{A}$ and $\mathcal{B}$ denoted by $\mathcal{A} \cap \mathcal{B}$ is defined by:

$$
\mu_{\mathcal{A} \cap \mathcal{B}}=\min \left(\mu_{\mathcal{A}}(x), \mu_{\mathcal{B}}(x)\right), x \in U .
$$

The union operation (3) of $\mathcal{A}$ and $\mathcal{B}$ denoted by $\mathcal{A} \cup \mathcal{B}$ is defined by: 


$$
\mu_{\mathcal{A} \cup \mathcal{B}}=\max \left(\mu_{\mathcal{A}}(x), \mu_{\mathcal{B}}(x)\right), x \in U .
$$

Membership function bell (4) is defined as:

$$
\operatorname{bell}(x ; a, b, c)=\frac{1}{1+\left|\frac{x-c}{a}\right|^{2 b}} \text {. }
$$

Fuzzy logic models use fuzzy sets to handle and describe vague and complex phenomena and use logical operations to reach the conclusion. Fuzzy arrays and fuzzy logic applied to control problems is related to a field of knowledge called Fuzzy Logic Control (FLC). It deals with control problems in an environment of uncertainty and precision. It is very effective when high accuracy is not required and the control object has variables available for measurement or estimation.

The diffuse control always involves the fuzzification process, which is performed always, it is the gateway to the diffuse inference system. It is a mathematical procedure in which an element of the universe of discourse (variable measured from the process) is converted into fuzzy set with a membership function.

Fuzzy controllers use rules, which combine one or more fuzzy sets of inputs called antecedents or premises and associate a fuzzy set of output called consequent or consequence. They involve fuzzy sets, fuzzy logic and fuzzy inference. These rules are called fuzzy rules, which are affirmations of the type IF-THEN. Fuzzy antecedent arrays are associated by blurred logical operations AND, OR, etc.

The rule used in this controller is Mamdami's (1975) fuzzy rule (5), which is a conjunction rule expressed by the operation $\wedge$ (minime); If $r_{k}$ is the conclusion or consequent and $p_{i}$ and $q_{j}$ are the premises or antecedents, then:

$$
\begin{aligned}
& \quad,\left(p_{i} \wedge q_{j}\right) \rightarrow r_{k}=\min \left(\mu_{\mathcal{A}_{i}}(x), \mu_{\mathcal{B}_{j}}(y), \mu_{\mathcal{C}_{k}}(z)\right) r_{k}=r_{i j}, \\
& \text { with } i=1,2, \ldots, n ; j=1,2, \ldots, m ; k=1,2, \ldots, l ; \mathrm{y}(x, y, z) \in U_{1} \times U_{2} \times \\
& U_{3} \text {. }
\end{aligned}
$$

When evaluating the rules, you get as many fuzzy sets as there are rules, to defuse it is necessary to group these sets, this stage is called aggregation and there are several criteria to carry out this step. One of the most frequently used criteria is to group the inferred sets by means of the operation $\vee$ (maxime).

Defuzzification is a mathematical process used to convert a fuzzy set into a real number. The fuzzy inference system obtains a conclusion from the input information, but it is in fuzzy terms. This conclusion or diffuse output is obtained by the stage fuzzy inference, it generates a fuzzy set but the output data of the system must be a real number and must be representative of the whole set obtained in the aggregation stage, that is why there are different methods of defuzzification and give different results, the "most common and widely used" is the gravity centroid. With the centroid defuzzification method, the fuzzy output is transformed into a real number which is the coordinate of the center of gravity (1.6) of a fuzzy set output and is given by equation (6):

$$
z_{C G}=\frac{\sum_{k=1}^{l} z_{k} \mu\left(z_{k}\right)}{\sum_{k=1}^{l} \mu\left(z_{k}\right)}
$$


Optimization of a Production Process from Demand and Number of Defective Units...

A genetic algorithm (GA) simulates some aspects of Darwin's evolutionary theory of species and the laws of Gregory Mendel's inheritance. The first genetic algorithm was developed by John Holland in the 1960s at the University of Michigan; the pseudocode of his work is listed below:

- Define an objective function.

- Random Generation of possible solutions (population).

- Encoding population.

- Evaluate the population, thus starting the i-generation.

- Select solutions to be reproduced.

- Crossover operation.

- Offspring mutation.

- Replace elements of the population of the i-generation with the best element-elements of 6 and 7 .

- Stop if the stop criterion is met, otherwise go to 4 .

Fuzzy logic and genetic algorithms are part of the techniques that fall under the concept of soft computing, a term coined by Lofti Zadeh in 1992. When there is synergy between the above techniques, it is called computational intelligence.

\section{Methodology}

To make the diffuse inference machine, information was collected from an expert in the administration of the process of interest, with respect to the levels of demand, defective units and increase and decrease of units in the production process that are taken into planning, for example for the set increased demand was considered the range of 20 to 30 units with value of belonging to 1 , from 10 to 20 units a linear function with value of membership 0 in 10 units and 1 in 20 units and from 30 to 40 units another linear function with membership value 1 in 30 units and 0 in 40 units. It is clearly seen that the belonging function of said set is trapezoidal, but to reduce the number of variables in the objective function of the genetic algorithm, a bell function was adjusted with domain equal to the interval [-50.50], which is the universe corresponding to the demand, and the function of belonged was defined by $\mu_{A}=\operatorname{bell}(x ; 10,4,25)$. Subsequently the same was done for each of the sets defined in the section. Then once having the defined sets, we continue to formulate the implications based on the experience of the manager or administrator of the process. The rule used in the controller is that of Mamdami, the aggregate was made through the maximum operation and the defuzzification by centroid.

In this work, the aim is not to minimize time directly, but to maintain an adequate level of production that will restore demand at a given time. The experience and knowledge of industrial engineers is of great importance in the construction of the model, since through them the possible alternatives that can be taken are considered.

The fuzzy models presented here have two input variables: The value of demand $\mathcal{D}$ for a product and the number of defective units $\mathcal{U}$ that are taken at the end of the 
production process. The output variable, the production action $(\mathcal{P})$, suggests elaborating more units or no action at some point.

The entries are modelled by set $\mathcal{D}$ with five terms:

$$
\mathcal{D}=\{S, D, E, A, C\},
$$

where $S:=$ decreasing, $D:=$ diminished, $E:=$ stable, $A:=$ augmented and $C:=$ increasing.

And the set $\mathcal{U}$ with four parameters:

$$
\mathcal{U}=\{N, P, R, B, X\},
$$

where $N:=$ low $P:=$ few $R:=$ average, $B:=$ several and $X:=$ exaggerated.

The output is given by $\mathcal{P}$ with four parameters:

$$
\mathcal{P}=\{O, A P, A M, A G\},
$$

where $O:=$ zero, $A P:=$ little augmented, $A M:=$ average augmented and $A G:=$ high augmented.

The universe considered in demand is $U_{\mathcal{D}}=\{a \in \mathbb{Z}:-50 \leq a \leq 50\}$, for the defective units is $U_{\mathcal{U}}=\{b \in \mathbb{Z}: 0 \leq b \leq 25\}$ and for the production is $U_{\mathcal{P}}=$ $\{c \in \mathbb{Z}: 0 \leq b \leq 40\}$. The membership functions for each set corresponding to $\mathcal{D}$ (Demand) are:

$$
\begin{array}{cc}
\mu_{S}=\operatorname{bell}(x ; 25,1,-50), & \mu_{D}= \\
& \operatorname{bell}(x ; 10,10,-15), \\
\mu_{A}=\operatorname{bell}(x ; 10,4,25), \quad \mu_{C}=\operatorname{bell}(x ; 20,1,50) .
\end{array}
$$

The membership functions for each set corresponding to $\mathcal{U}$ (Defective units) are:

$$
\begin{array}{ll}
\mu_{N}=\operatorname{bell}(x ; 0.9,1,0), & \mu_{P}=\operatorname{bell}(x ; 4,11,7.5), \quad \mu_{R}=\operatorname{bell}(x ; 5,5,9), \\
\mu_{B}= & \mu_{X}=\operatorname{bell}(x ; 1,1,25) .
\end{array}
$$

The membership functions for each set corresponding to $\mathcal{P}$ (Production) are:

$$
\begin{aligned}
& \mu_{O}=\operatorname{bell}(x ; 1,1,0), \quad \mu_{A P}=\operatorname{bell}(x ; 4,9,5), \quad \mu_{A M}=\operatorname{bell}(x ; 8,8,16), \\
& \mu_{A G}=\operatorname{bell}(x ; 8,14,35) .
\end{aligned}
$$

The number of rules obtained from the expert information is 25 (table 1), these rules were developed from information collected during one year on a production line and these conclude in the terms of departure. 
Table 1. Rules given by expert.

\begin{tabular}{cccccc}
\hline & $\boldsymbol{N}$ & $\boldsymbol{P}$ & $\boldsymbol{R}$ & $\boldsymbol{B}$ & $\boldsymbol{X}$ \\
\hline $\boldsymbol{S}$ & $O$ & $O$ & $O$ & $O$ & $A P$ \\
\hline $\boldsymbol{D}$ & $O$ & $O$ & $O$ & $A P$ & $A M$ \\
\hline $\boldsymbol{E}$ & $O$ & $O$ & $O$ & $A M$ & $A M$ \\
\hline $\boldsymbol{A}$ & $O$ & $A P$ & $A M$ & $A M$ & $A G$ \\
\hline $\boldsymbol{C}$ & $A P$ & $A P$ & $A M$ & $A M$ & $A G$ \\
\hline
\end{tabular}

The following are some rules that lead to the production action according to table 1. Rule given by row 1 and column 1 of table (1): If $\mathcal{D}$ is decreasing $(S)$ and $\mathcal{U}$ is low $(\boldsymbol{N})$, then nothing must be done in production $(\boldsymbol{O})$.

Rule given by row 4 and column 3 of table (1): If $\mathcal{D}$ is augmented $(\boldsymbol{A})$ and $\mathcal{U}$ is average $(\boldsymbol{R})$, then a medium increase in production must be made $(\boldsymbol{A} \boldsymbol{M})$.

For the objective function (fitness) of the genetic algorithm tuned by the fuzzy inference machine, the vector with the initial parameters equation (7) of the bell membership functions is given by an expert:

$A=\left[\begin{array}{llllll}25 & -50 & 10 & 10 & -157.53010425201500 .9104117 .555941613 .51125\end{array}\right.$ 110495881681435 ]

The objective function (equation (8)) is:

$$
y=\frac{1}{42} \sum_{i=1}^{42}\left|x_{i}-A_{i}\right|
$$

Where $A_{i}$ is the i-element of vector $A$. The objective is to minimize the function given in equation (8).

Since the problem to be optimized is of dimension 42, an initial population of 5000 individuals is used, each with 672 alleles, in which every 16 alleles form a chain of bits that represent a real number and a resolution of 1000 is used.

The selection method is by tournament, the tournament size applied here is 10 , the number of crossover points is 5 , the mutation percentage is $4 \%$ and 5000 generations are used.

\section{Results}

The comparative between the results obtained with genetic algorithm tuned values and the expert recommended system is shown below.

The table that shows the parameters given by the expert and the genetic algorithm of the membership functions corresponding to the demand are shown in table 2 .

Table 2. Demand table.

\begin{tabular}{ccc}
\hline Demand & Expert & Genetic Algorithm \\
\hline Decreasing & $\mu_{S}=\operatorname{bell}(x ; 25,1,-50)$ & $\mu_{S}=\operatorname{bell}(x ; 24.00,1.03,-32.76)$ \\
\hline
\end{tabular}


Christian A. Mejía Ramírez, Martin Montes Rivera

\begin{tabular}{clc}
\hline Diminished & $\begin{array}{c}\mu_{D} \\
=\operatorname{bell}(x ; 10,10,-15)\end{array}$ & $\mu_{D}=\operatorname{bell}(x ; 10.01,9.98,-16.38)$ \\
\hline Stable & $\mu_{E}=\operatorname{bell}(x ; 7.5,3,0)$ & $\mu_{E}=\operatorname{bell}(x ; 7.17,-0.004,-0.008)$ \\
\hline Aumented & $\mu_{A}=\operatorname{bell}(x ; 10,4,25)$ & $\mu_{A}=\operatorname{bell}(x ; 10.00,4.00,24.57)$ \\
\hline Increasing & $\mu_{S}=\operatorname{bell}(x ; 20,1,50)$ & $\mu_{S}=\operatorname{bell}(x ; 16.38,0.98,32.77)$ \\
\hline
\end{tabular}

The table that shows the parameters given by the expert and the genetic algorithm of the belonging functions corresponding to the defective units is show in table 3 .

Table 3. Defective units.

\begin{tabular}{cccc}
\hline $\begin{array}{c}\text { Defective } \\
\text { Units }\end{array}$ & \multicolumn{1}{c}{ Expert } & Genetic Algorithm \\
\hline Low & $\mu_{N}=\operatorname{bell}(x ; 0.9,1,0)$ & $\begin{array}{l}\mu_{N} \\
=\operatorname{bell}(x ;-0.0005,1.0085,0.0045)\end{array}$ \\
\hline Few & $\mu_{P}=\operatorname{bell}(x ; 4,11,7.5)$ & $\mu_{P}=\operatorname{bell}(x ; 4.10,11.00,7.5)$ \\
\hline Average & $\mu_{R}=\operatorname{bell}(x ; 5,5,9)$ & $\mu_{R}=\operatorname{bell}(x ; 4.09,8.20,8.96)$ \\
\hline Several & $\mu_{B}$ & $\mu_{B}$ & $=\operatorname{bell}(x ;-0.0015,16.3925,13.5)$ \\
& $=\operatorname{bell}(x ; 4,16,13.5)$ & $\mu_{X}=$ \\
Exaggerated & $\mu_{X}=\operatorname{bell}(x ; 1,1,25)$ & $\operatorname{bell}(x ;-0.0045,0.9995,24.58)$ \\
\hline
\end{tabular}

The table that shows the parameters given by the expert and by the genetic algorithm of the belonging functions corresponding to production is shown in table 4 .

Table 4. Production table.

\begin{tabular}{lcc}
\hline \multicolumn{1}{c}{ Production } & Expert & \multicolumn{1}{c}{ Genetic Algorithm } \\
\hline Zero & $\mu_{O}=\operatorname{bell}(x ; 1,1,0)$ & $\begin{array}{l}\mu_{O} \\
=\operatorname{bell}(x ; 1.03,-0.0005,0.0005)\end{array}$ \\
\hline Little Augmented & $\mu_{A P}=\operatorname{bell}(x ; 4,9,5)$ & $\mu_{A P}=\operatorname{bell}(x ; 3.96,8.19,5.12)$ \\
\hline $\begin{array}{l}\text { Average } \\
\text { Augmented }\end{array}$ & $\mu_{A M}=\operatorname{bell}(x ; 8,8,16)$ & $\mu_{A M}=\operatorname{bell}(x ; 8.20,8.19,16.39)$ \\
\hline High Augmented & $\mu_{A G}=\operatorname{bell}(x ; 8,14,35)$ & $\mu_{A G}=\operatorname{bell}(x ; 8.00,14.02,32.76)$ \\
\hline
\end{tabular}

The minimum value of the target function released by the genetic algorithm is 1.4136 , which is the average error between the obtained value and the expert vector.

The functions tuned by the genetic algorithm are shown in the figure 1, this graph shows the tuning (red dotted lines), of the functions of belonging (blue continuous lines), of the diffuse sets corresponding to demand, defective units and action in production. 

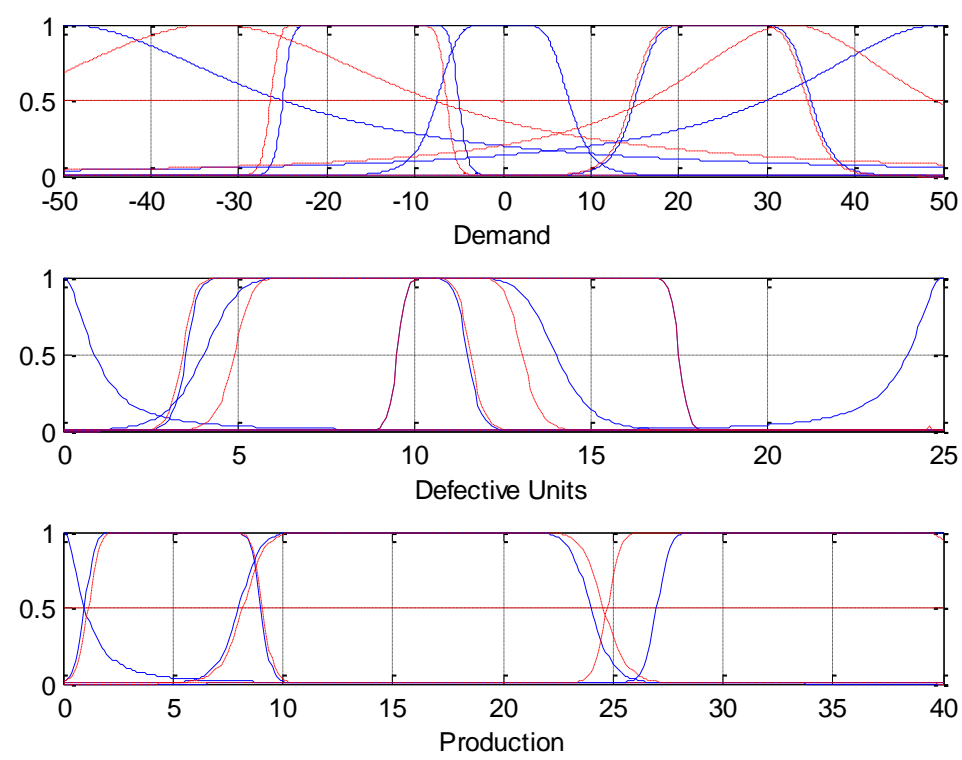

Fig. 1. Graph with comparative for demand, defective units and production sets with expert suggestion and Mamdani fuzzy logic system tuned with GA.

\section{Conclusion}

In this work is presented a self-tuned Mamdani fuzzy logic system by using GA, this fuzzy logic implementation it is applied for optimizing a production process based on demand required and the number of defective units, the proposed work achieves similar results to those suggested by a production expert.

One of the objectives of production planning is to anticipate the future for the efficient and effective completion of pre-established goals in planning. The objective of production planning is to make optimal use of material and financial resources to: a) meet demand, b) seize opportunities that may arise in the market and c) avoid the undesirable. However, in this process we cannot have a fixed demand nor avoid the undesirable, as in the case of having defective units, that is why experience in the processes can generate basic and efficient rules to have a control in the process and with them satisfy the demand and maximize the resources efficiency, in order to avoid the undesirable.

By means of the fuzzy control it is possible to make a production planning tuned with a GA, since when entering a value in the level of demand and in the level of defective units, the action in the production is generated. The parameters of the membership functions to the fuzzy sets corresponding to the action in the production of the table (4) shows that the GA is capable of tuning four of the output functions very similarly to those given by the expert; however, it cannot be said that the non-similar 
functions thrown by the algorithm (Fig. 1), are not adequate, since expert system and genetic algorithm data differ only by an average 1.4136 units.

With regard to future work, we will seek to implement another objective function of the genetic algorithms to tune belongings function in such a way that the production time of the process is estimated. It will also be sought to implement these techniques to the inventory control systems of a pharmacy of public hospital.

\section{References}

1. Bojadziev, G., Bojadziev, M.: Fuzzy Logic for Business, Finance and Management. World Scientific, Singapore (2007)

2. Ross, T.J.: Fuzzy logic with engineering applications. Wiley (2004)

3. Sivanandam, S.N., Sumathi, S., Deepa S.N.: Introduction to Fuzzy Logic using MATLAB. Springer (2007)

4. Nguyen-Hung, T., Walker-Elbert, A.: A First Course in Fuzzy Logic. Champan \& Hall (2006)

5. Bramlette, M.F.: Initialization, Mutation and Selection Methods in Genetic Algorithms for Function Optimization. Proceedings of Fourth International Conference on Genetic Algorithms, pp. 100-107 (1991)

6. Holland, J.H. Adaptation in Natural and Artificial Systems. Cambridge (1992)

7. Peña-Reyes, C.A.: Coevolutionary Fuzzy Modeling. Lecture Notes in Computer Science. 3204, Springer (2004)

8. Rahnamayan, S., Tizhoosh, H.R., Salama, M.M.A.: A Novel Population Initialization Method for Accelerating Evolutionary Algorithms. Computers and Mathematics with Applications, 53(10), pp: 1605-1614 (2007)

9. Wang, L.X.: A Course in Fuzzy Systems and Control. Prentice-Hall (1997)

10. Yager, R.R., Zadeh, L.A.: Fuzzy Sets, Neural Networks, and Soft Computing. Van Nostrand Reinhold (1994)

11. Cengiz, K., Murat, G., Özgür, K.: Applications of Fuzzy Sets in Industrial Engineering: A Topical Classification. StudFuzz, 201, pp. 1-55 (2006)

12. Skalna, I., Rebiasz, B., et al.: Advances in Fuzzy Decision Making. Studies in Fuzziness and Soft Computing, Springer (2015)

13. Kahraman, C., Tolga, A.: Fuzzy Investment Planning and Analyses in Production Systems. STUDFUZZ, 252, pp. 279-298 (2010)

14. Gladkov, L.A. et al.: Hybrid Fuzzy Algorithm for Solving Operational Production Planning Problems. Advances in Intelligent Systems and Computing 573 (2017)

15. Verma, R. et al.: Limits, Compression, Generalized Model and Heuristics. Computación y Sistemas, 21(4), pp. 787-798 (2017)

16. Smailovic, J. et al.: Automatic Analysis of Annual Financial Reports: A Case of Study. Computación y Sistemas, 21(4), pp. 809-818 (2007)

17. Cruz, E. et al.: Simulation and Application of Algorithms CVRP to Optimize the Transport of Mineral Metallic and Nonmetallic by Rail to Export Level, Research in Computing Science 122, pp. 77-88 (2010)

18. Briones, G.E. et al.: Design of Technology Management Infrastructure for Large Volumes of Data in Intelligent Power Network. Research in Computing Science 122, pp. 113$126(2016)$

19. Souverville, S. et al.: Fuzzy Logic Applied to Improvement of Image Resolution using Gaussian Membership Functions, Research in Computing Science 102, pp. 77-88 (2015) 